\title{
ON A THEOREM OF BECK
}

\author{
by K. F. ROTH
}

Dedicated to Professor R. A. Rankin on the occasion of his 70 th birthday

1. Introduction. Let $T(N)$ be the least integer such that one can assign \pm 1 's to any $N$ points in the unit square so that the sum of these values in any rectangle with sides parallel to those of the square have absolute value at most $T(N)$. In [1] Beck showed, among other results, that (for $N \geqslant 2$ )

$$
\log N \ll T(N) \ll(\log N)^{4} .
$$

The lower bound asserts the existence of a set $\mathscr{P}$ of $N$ points $\mathbf{p}$ in the square such that if "weights" $\lambda(\mathbf{p})$, where $\lambda(\mathbf{p})= \pm 1$, are freely assigned to the points $\mathbf{p}$ of $\mathscr{P}$, there is a rectangle $B$ with sides parallel to those of the square such that

$$
\left|\sum_{\mathbf{p} \in \mathscr{P} \cap \mathbf{B}} \lambda(\mathbf{p})\right| \gg(\log N) N^{-1} \sum_{\mathbf{p} \in \mathscr{P}}|\lambda(\mathbf{p})| .
$$

Beck deduced the existence of such a set $\mathscr{P}$ from a theorem of Wolfgang Schmidt [2]. In the present paper we use a direct application of a variant of Halasz's method [4] for proving Schmidt's theorem to show that a certain type of set, which is easy to construct, has the above property given an entirely unrestricted set of real weights $\lambda(\mathbf{p})$.

We use $U$ to denote the unit interval $(0,1]$. If $\alpha=\left(\alpha_{1}, \alpha_{2}\right), \boldsymbol{\beta}=\left(\beta_{1}, \beta_{2}\right)$ are two points in the unit square $U^{2}$, the sum $\boldsymbol{\alpha}+\boldsymbol{\beta}$ denotes the point in $U^{2}$ obtained by interpreting the vector addition modulo 1 in each coordinate.

Let $\mathscr{P}$ be a finite set of points in $U^{2}$ and suppose that a real number $\lambda(\mathbf{p})$ is assigned to each $\mathbf{p}$ in $\mathscr{P}$. We write

$$
Z(\boldsymbol{\alpha} ; \mathbf{x})=\sum_{\mathbf{p},(1.4)} \lambda(\mathbf{p})
$$

where the summation is over those $\mathbf{p}$ of $\mathscr{P}$ for which

$$
\boldsymbol{\alpha}+\mathbf{p} \text { lies in the rectangle }\left(0, x_{1}\right] \times\left(0, x_{2}\right] .
$$

For $\mathbf{v}=\left(v_{1}, v_{2}\right)$ we write $d(\mathbf{v})=\left\|v_{1}\right\| .\left\|v_{2}\right\|$, where $\|\beta\|$ is the distance of $\beta$ from the nearest integer.

Separation Property. We say that $\mathscr{P}$ is well-separated with respect to the nonnegative integer $n$ if, subject to the additional condition $\mathbf{p}^{\prime} \neq \mathbf{p}^{\prime \prime}$,

$$
\inf _{\mathbf{p}^{\prime} \in \mathscr{P}, \mathbf{p}^{\prime \prime} \in \mathscr{P}} d\left(\mathbf{p}^{\prime}-\mathbf{p}^{\prime \prime}\right) \geqslant 2^{-n} .
$$

We shall prove the following result.

Glasgow Math. J. 27 (1985) 195-201 . 
THEOREM. If $\mathscr{P}$ is well-separated with respect to $n$, then there exist $\mathbf{\alpha}, \mathbf{x}$ such that

$$
|Z(\boldsymbol{\alpha} ; \mathbf{x})| \geqslant(n+1) 2^{-n-4} \sum_{\mathbf{p} \in \mathscr{P}}|\lambda(\mathbf{p})| .
$$

We note that, reserving $J$ for subintervals of $U$ of type $(0, x]$, the characteristic function of the translated interval $-\alpha+J$ modulo 1 can (for given $J$ ) be represented in the form $\pm J^{(1)}(v) \pm J^{(2)}(v) \pm J^{(3)}(v)$ where $J^{(\nu)}(v)$ is the characteristic function on interval $\boldsymbol{J}^{(\nu)}$. Thus (1.6) implies the existence of an $\mathbf{x}^{*}$ in $U^{2}$ such that

$$
\left|Z\left(\mathbf{0} ; \mathbf{x}^{*}\right)\right| \geqslant 3^{-2}(n+1) 2^{-n-4} \sum_{\mathbf{p} \in \mathscr{P}}|\lambda(\mathbf{p})| .
$$

To ensure that the right hand side of (1.6) is $\gg$ the right hand side of (1.2) we need to construct a set $\mathscr{P}$ of $N$ points which satisfies the condition (1.5) for some $n \ll \log N$. For this purpose we can, for example, make use of the well-known fact that if $\theta$ is an irrational number whose continued fraction has bounded partial quotients, then

$$
m\|\theta m\|>c>0 \quad(m=1,2, \ldots),
$$

where $c=c(\theta)$ depends only on $\theta$. Thus the set

$$
\left(\left\{\theta m^{\prime}\right\}, \frac{1}{2} m^{\prime} N^{-1}\right) \quad\left(m^{\prime}=0,1, \ldots, N-1\right)
$$

has the desired property (where $\{\phi\}$ denotes the fractional part of $\phi$ ).

In fact it is not hard to construct sets with the desired separation property without the use of any results concerning Diophantine approximation, although we do not do so here.

The well-separation hypothesis is much more stringent than we require to obtain a result of type (1.6) to within an absolute constant: the hypothesis can be relaxed in a number of respects. We have chosen the strong form of the hypothesis partly because in two dimensions it is easy to construct sets with this form of the hypothesis, but mainly to present the method in its simplest form.

A particularly interesting feature of this variant of the Halász method is that there are no error terms to estimate; here all errors cancel.

2. Notation. We adopt a system of notation very closely related to that introduced by Wolfgang Schmidt [3]. However, for technical reasons, we work with intervals closed at the right hand end instead of at the left hand end as has been previously customary. This change affects only sets of measure zero and is therefore inconsequential.

Every $x$ in $U$ has a unique representation

$$
x=\sum_{j=1}^{\infty} \beta_{j}(x) 2^{-j}
$$

where the digits $\beta_{i}(x)$ are 0 or 1 , and where the set of $j$ with $\beta_{j}(x)=1$ is infinite. The functions

$$
R_{r}(x)=(-1)^{\beta_{r+1}(x)} \quad(r=0,1, \ldots)
$$

are essentially the Rademacher functions. 
An $r$-interval will be an interval

$$
m 2^{-r}<x \leqslant(m+1) 2^{-r}
$$

where $m$ is an integer in $0 \leqslant m<2^{r}$. A $C_{r}$-function, where $r$ is a non-negative integer, is a function $C_{r}(x)$ which is constant on every $r$-interval. If $C_{r}(x)$ is a $C_{r}$-function, then the product $C_{r}(x) R_{r}(x)$ is said to be a modified $R_{r}$-function. Clearly the following holds.

LEMMA 1. If $M_{r}(x)$ is a modified $R_{r}$-function, then

$$
\int_{U} M_{r}(x)=0
$$

We now consider $k$-dimensional space. Although in the present paper we will have $k=2$, we formulate definitions for general $k$ for use in subsequent work.

Given a $k$-tuple $\mathbf{r}=\left(r_{1}, \ldots, r_{k}\right)$ of non-negative integers, put

For $\mathbf{x}$ in $U^{k}$, write

$$
|\mathbf{r}|=r_{1}+\ldots+r_{k} .
$$

$$
R_{\mathbf{r}}(\mathbf{x})=R_{r_{1}}\left(x_{1}\right) \ldots R_{r_{k}}\left(x_{k}\right) .
$$

An $\mathbf{r}$-box is defined as a box $I_{1} \times I_{2} \times \ldots \times I_{k}$ where $I_{j}$ is an $r_{j}$-interval for $j=1,2, \ldots, k$. A $C_{\mathbf{r}}$-function is a function $C_{\mathbf{r}}(\mathbf{x})$ on $U^{k}$ which is constant on every $r$-box. Note that, for given $\mathbf{r}$, the set of $C_{\mathbf{r}}$ functions is closed under multiplication. A modified $R_{\mathbf{r}}$-function is a function of type $C_{\mathbf{r}}(\mathbf{x}) R_{\mathbf{r}}(\mathbf{x})$ where $C_{\mathbf{r}}(\mathbf{x})$ is a $C_{\mathbf{r}}$-function. The following lemma is trivial.

LEMMA 2. For given $x_{1}, \ldots, x_{\mathbf{i}-1}, x_{j+1}, \ldots, x_{k}$, a modified $R_{\mathbf{r}}$-function is a modified $R_{j}$-function in the variable $x_{j}$.

3. Outline of the proof of the theorem. We may suppose without loss of generality that

$$
\sum_{\mathbf{p} \in \mathscr{P}} \lambda(\mathbf{p}) \geqslant 0
$$

Define $e(\mathbf{p})$ to be +1 or -1 according as $\lambda(\mathbf{p}) \geqslant 0$ or $\lambda(\mathbf{p})<0$. Then the numbers $e(\mathbf{p})$ satisfy

$$
(e(\mathbf{p}))^{2}=1, \quad e(\mathbf{p}) \lambda(\mathbf{p})=|\lambda(\mathbf{p})| \quad(\mathbf{p} \in \mathscr{P}) .
$$

Henceforth we assume $k=2$ when using the notation of $\$ 2$.

Corresponding to each $\mathbf{r}$ we denote by $\mathscr{A}_{\mathbf{r}}$ the set of all $\mathbf{r}$-boxes. We use $B(\mathbf{x})$ to denote the characteristic function of the box $B$, so that

and define

$$
B(\mathbf{x})=\left\{\begin{array}{lll}
1 & \text { if } & \mathbf{x} \in B \\
0 & \text { if } & \mathbf{x} \notin B
\end{array}\right.
$$

$$
g_{\mathbf{r}}(\boldsymbol{\alpha} ; \mathbf{x})=R_{\mathbf{r}}(\mathbf{x}) \sum_{\mathbf{p} \in \mathscr{P}} e(\mathbf{p}) \sum_{B \in \mathscr{A}_{\mathbf{r}}} B(\boldsymbol{\alpha}+\mathbf{p}) B(\mathbf{x})
$$


Let $\mathscr{M}=\mathscr{M}(n)$ be the set of all $\mathbf{r}$ satisfying $|\mathbf{r}|=n$ (that is $r_{1}+r_{2}=n$, where $n$ is the parameter featuring in the "separation property" $(1.5)$ ), and let $\mathbb{B}=\mathbb{B}(n)$ to be the set of all subsets $\mathscr{S}$ of $\mathcal{M}$ (including the empty subset). We shall make use of the fact that there are $n+1$ sets $\mathscr{S}$ consisting of precisely one element.

The relevance of the "separation property" is that it ensures that, for given $\boldsymbol{\alpha}$, an $\boldsymbol{r}$-box with $\mathbf{r} \in \mathcal{M}$ contains at most one point $\boldsymbol{\alpha}+\mathbf{p}$. In particular,

$$
\mathbf{r} \in \mathcal{M} \text { implies } 1+\mathrm{g}_{\mathbf{r}}(\boldsymbol{\alpha} ; \mathbf{x}) \geqslant 0 .
$$

Using the key idea of Halász [4], we define

where

$$
F(\boldsymbol{\alpha} ; \mathbf{x})=\prod_{\mathbf{r} \in \mathcal{M}}\left(1+\mathrm{g}_{\mathbf{r}}(\boldsymbol{\alpha} ; \mathbf{x})\right)=\sum_{\mathscr{S} \in \mathcal{G}} \prod_{\mathscr{S}}(\boldsymbol{\alpha} ; \mathbf{x}),
$$

$$
\prod_{\mathscr{S}}(\boldsymbol{\alpha} ; \mathbf{x})=\prod_{\mathbf{r} \in \mathscr{S}} \mathrm{g}_{\mathbf{r}}(\boldsymbol{\alpha} ; \mathbf{x})
$$

In the final section we shall establish the following two lemmas.

LEMMA 3. Suppose $\mathscr{S}$ is non-empty. Then, for every $\alpha$,

$$
\int_{U^{2}} \prod_{\mathscr{S}}(\boldsymbol{\alpha} ; \mathbf{x}) d \mathbf{x}=0
$$

LEMmA 4. Let $l=\operatorname{card} \mathscr{S}$ be the number of elements of $\mathscr{S}$. Then, writing

we have

$$
I_{\mathscr{S}}=\int_{U^{2}} d \boldsymbol{\alpha} \int_{U^{2}} \prod_{\mathscr{S}}(\boldsymbol{\alpha} ; \mathbf{x}) Z(\boldsymbol{\alpha} ; \mathbf{x}) d \mathbf{x}
$$

$$
I_{\mathscr{P}}= \begin{cases}2^{-n-4} \sum_{\mathbf{p} \in \mathscr{P}}|\lambda(\mathbf{p})| & \text { if } \quad l=1, \\ 0 & \text { if } \quad l \geqslant 2 .\end{cases}
$$

Despite our somewhat different auxiliary functions $g_{r}$, Lemma 3 and its proof are in essence exactly the same as for the corresponding result in Halász's work. On the other hand, Lemma 4 embodies a new feature of the present variant of Halász's method. In Halász's work the terms corresponding to card $\mathscr{S} \geqslant 2$ gave rise to an error which needed to be estimated.

We now deduce the theorem from Lemmas 3 and 4. By (3.4), (3.5) and Lemma 3,

$$
\int_{U^{2}} d \boldsymbol{\alpha} \int_{U^{2}}|F(\boldsymbol{\alpha} ; \mathbf{x})| d \mathbf{x}=\int_{U^{2}} d \boldsymbol{\alpha} \int_{U^{2}} F(\boldsymbol{\alpha} ; \mathbf{x}) d \mathbf{x}=1 .
$$

On the other hand since for each $\mathbf{x}$,

$$
\int_{U^{2}} Z(\boldsymbol{\alpha} ; \mathbf{x}) d \mathbf{\alpha}=x_{1} x_{2} \sum_{\mathbf{p} \in \mathscr{P}} \lambda(\mathbf{p})
$$


it follows from (3.5), (3.1) and Lemma 4 that

$$
\int_{U^{2}} d \boldsymbol{\alpha} \int_{U^{2}} F(\boldsymbol{\alpha} ; \mathbf{x}) Z(\boldsymbol{\alpha} ; \mathbf{x}) d \mathbf{x} \geqslant(n+1) 2^{-n-4} \sum_{\mathbf{p} \in \mathscr{P}}|\lambda(\mathbf{p})| .
$$

Comparison of (3.10) and (3.11) immediately yields the theorem. It now only remains to prove Lemmas 3 and 4.

4. Completion of the proof. We consider a fixed non-empty set $\mathscr{S}$. Suppose this $\mathscr{S}$ consists of the $l$ vectors

$$
\mathbf{r}_{1}=\left(r_{11}, r_{12}\right), \quad \mathbf{r}_{2}=\left(r_{21}, r_{22}\right), \ldots, \mathbf{r}_{1}=\left(r_{11}, r_{12}\right) .
$$

Since $r_{i 1}+r_{j 2}=n$ for each $j$, we may suppose

$$
r_{11}<r_{21}<\ldots<r_{11}
$$

We write $t=\left(t_{1}, t_{2}\right)=\left(r_{11}, n-r_{11}\right)$ so that

$$
t_{i}=\max \left(r_{1 i}, r_{2 i}, \ldots, r_{i i}\right) \text { for } i=1,2 .
$$

It follows from the "separation property" of $\mathscr{P}$ that

$$
\begin{gathered}
\text { if } B \text { is a } \mathbf{t} \text {-box then } B(\boldsymbol{\alpha}+\mathbf{p})=1 \text { implies } \\
B(\mathbf{x}) g_{\mathbf{r}_{i}}(\boldsymbol{\alpha} ; \mathbf{x})=e(\mathbf{p}) B(\mathbf{x}) R_{\mathbf{r}_{i}}(\mathbf{x}) \quad(j=1,2, \ldots l) .
\end{gathered}
$$

Thus

$$
\prod_{\mathscr{S}}(\boldsymbol{\alpha} ; \mathbf{x})=C_{\mathbf{t}}^{*}(\boldsymbol{\alpha} ; \mathbf{x}) \prod_{\mathbf{r} \in \mathscr{S}} R_{\mathbf{r}}(\mathbf{x}),
$$

where, for each $\boldsymbol{\alpha}, C_{\mathbb{t}}^{*}$ is a $C_{\mathbf{t}}$ function such that

if $B$ is a $t$-box then $B(\alpha+p)=1$ implies

$$
B(\mathbf{x}) C_{\mathbf{i}}^{*}(\boldsymbol{\alpha} ; \mathbf{x})=(e(\mathbf{p}))^{\prime} B(\mathbf{x}) .
$$

Now

where

$$
\prod_{\mathbf{r} \in \mathscr{S}} R_{\mathbf{r}}(\mathbf{x})=Q(\mathbf{x}) R_{\mathbf{t}}(\mathbf{x})
$$

$$
Q(\mathbf{x})=\left(\prod_{i=1}^{l-1} R_{i 1}\left(x_{1}\right)\right)\left(\prod_{i=2}^{l} R_{j 2}\left(x_{2}\right)\right)
$$

Note that

$$
Q(\mathbf{x}) \text { is a } C_{\mathbf{t}} \text { function, }
$$

and (by Lemmas 1 and 2), since $Q(\mathbf{x})$ is also a modified two dimensional $R$-function when $l \geqslant 2$,

$$
\int_{U^{2}} Q(\mathbf{x}) d \mathbf{x}=\left\{\begin{array}{lll}
1 & \text { if } & l=1 \\
0 & \text { if } & l \geqslant 2
\end{array}\right.
$$


We also note that (4.5), (4.6) and (4.7) yield

if $B$ is a $\mathbf{t}$-box then $B(\boldsymbol{\alpha}+\mathbf{p})=1$ implies

$$
B(\mathbf{x}) \prod_{\mathscr{\rho}}(\boldsymbol{\alpha} ; \mathbf{x})=(e(\mathbf{p}))^{l} B(\mathbf{x}) Q(\mathbf{x}) R_{\mathbf{t}}(\mathbf{x}) .
$$

Since (4.7) is a modified $\boldsymbol{R}_{\mathbf{t}}$-function, so also is the function (4.5) for each fixed $\boldsymbol{\alpha}$. Thus (3.7) holds, and Lemma 3 is proved. It remains to prove Lemma 4 by evaluating the $I_{\mathscr{S}}$ defined in (3.8).

Let $B$ be a fixed $\mathbf{t}$-box. For a fixed value of $\boldsymbol{\alpha}$, we consider the integral

$$
I_{\mathscr{S}, B}(\boldsymbol{\alpha})=\int_{\mathbf{B}} \prod_{\mathscr{S}}(\boldsymbol{\alpha} ; \mathbf{x}) Z(\mathbf{\alpha} ; \mathbf{x}) d \mathbf{x}
$$

Since (4.5) is a modified $R_{1}$ function, we first evaluate

$$
\int_{B} R_{\mathbf{r}}(\mathbf{x}) Z(\boldsymbol{\alpha} ; \mathbf{x}) d \mathbf{x} \text {. }
$$

Suppose the t-box $B$ is given by $m_{i} 2^{-t_{i}}<x_{i} \leqslant\left(m_{i}+1\right) 2^{-t_{i}}(i=1,2)$ and let $B^{\prime}$ be the (smaller) box $0<x_{i} \leqslant 2^{-t_{i}-1}(i=1,2)$. Then the integral (4.12) is

$$
\int_{B^{\prime}} \sum_{\varepsilon_{1}=0}^{1} \sum_{\varepsilon_{2}=0}^{1}(-1)^{\varepsilon_{1}+\varepsilon_{2}} Z\left(\alpha ; \mathbf{m}+\mathbf{y}+\left(\varepsilon_{1} 2^{-t_{1}-1}, \varepsilon_{2} 2^{-t_{2}-1}\right)\right) d \mathbf{y}
$$

and here the integrand represents

$$
\sum_{\mathbf{p} \in \mathscr{P}} \lambda(\mathbf{p}) B^{\prime}(\boldsymbol{\alpha}+\mathbf{p}-\mathbf{m}-\mathbf{y})
$$

namely the sum of the weights $\lambda(\mathbf{p})$ corresponding to points $\mathbf{p}$ for which $\boldsymbol{\alpha}+\mathbf{p}$ lies in the box $m_{i}+y_{i}<x_{i} \leqslant m_{i}+y_{i}+2^{-t_{i}-1}(i=1,2)$. This latter box is contained in $B$ for $\mathbf{y} \in B^{\prime}$ so that unless $\boldsymbol{\alpha}+\mathbf{p} \in B$, the summand in (4.13) vanishes for all $\mathbf{y}$ in $\boldsymbol{B}^{\prime}$. Thus the integral (4.12) is equal to

$$
\sum_{\mathbf{p} \in \mathscr{P}} \lambda(\mathbf{p}) \int_{B^{\prime}} B^{\prime}(\boldsymbol{\alpha}+\mathbf{p}-\mathbf{m}-\mathbf{y}) d \mathbf{y},
$$

and only terms with $\boldsymbol{\alpha}+\mathbf{p} \in B$ can contribute to this sum. In fact there is at most one such term, in view of the "separation property" of $\mathscr{P}$.

If the point $\boldsymbol{\alpha}+\mathbf{p}^{*}$ lies in $B$, then (4.10) is applicable and

$$
I_{\mathscr{S}, \mathrm{B}}(\boldsymbol{\alpha})=\left(e\left(\mathbf{p}^{*}\right)\right)^{l} q(B) \int_{\mathrm{B}} R_{\mathbf{t}}(\mathbf{x}) Z(\boldsymbol{\alpha} ; \mathbf{x}) d \mathbf{x},
$$

where $q(B)$ is the constant value of $Q(\mathbf{x})$ in $B$ : note that

$$
q(B)=(V(B))^{-1} \int_{B} Q(\mathbf{x}) d \mathbf{x}
$$

where $V(B)$ is the volume of $B$. If there is no $\mathbf{p}$ such that $\boldsymbol{\alpha}+\mathbf{p}$ lies in $B$ then (4.12) 
vanishes, and hence (recalling that (4.5) is a modified $R_{t}$ function) the integral (4.11) also vanishes. In either event, we have

Since

$$
I_{\mathscr{P}, B}(\boldsymbol{\alpha})=q(B) \sum_{\mathbf{p} \in \mathscr{P}}|\lambda(\mathbf{p})|(e(\mathbf{p}))^{l-1} \int_{\mathbf{B}^{\prime}} B^{\prime}(\boldsymbol{\alpha}+\mathbf{p}+\mathbf{m}-\mathbf{y}) d \mathbf{y}
$$

$$
\int_{U^{2}} B^{\prime}(\boldsymbol{\alpha}+\mathbf{p}+\mathbf{m}-\mathbf{y}) d \boldsymbol{\alpha}=V\left(B^{\prime}\right)=\frac{1}{4} V(B)=2^{-n-2}
$$

when $l=1$ it follows from (4.14) that

$$
\int_{U^{2}} I_{\mathscr{S}, \mathbf{B}}(\boldsymbol{\alpha}) d \boldsymbol{\alpha}=2^{-n-4} \sum_{\mathbf{p} \in \mathscr{P}}|\lambda(\mathbf{p})|(\mathrm{e}(\mathbf{p}))^{l-1} \int_{B} Q(\mathbf{x}) d \mathbf{x} .
$$

On summing over all boxes of type $B_{1}$, the assertion (3.9) of Lemma 4 follows from (4.9).

\section{REFERENCES}

1. József Beck, Balanced Two-Colorings of Finite Sets in the Square I, Combinatorica 1 (1981), 327-335.

2. W. M. Schmidt, Irregularities of Distribution VII, Acta Arith., 21 (1972), 45-50.

3. W. M. Schmidt, Irregularities of Distribution X, Number Theory and Algebra (ed. H. Zassenhaus, Academic Press, 1977), 311-329.

4. G. Halász, On Roth's method in the theory of irregularities of point distributions, Recent Progress in Analytic Number Theory, Vol. 2 (eds. H. Halberstam and C. Hooley, Academic Press, 1981), 79-94.

Department of Mathematics

IMPERIAL COLlEgE OF SCIENCE AND TECHNOLOGY

179-180 QUEEN'S GATE

LONDON

SW7 2BZ 